Revista Universo Contábil, ISSN 1809-3337

FURB, v. 7, n.1, p. 106-121, jan./mar., 2011

doi:10.4270/ruc.2011107

Disponível em www.furb.br/universocontabil

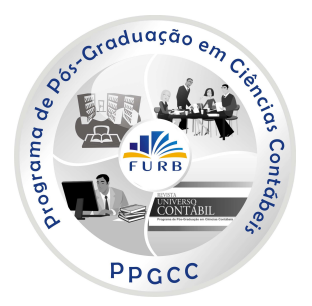

\title{
UNA APROXIMACIÓN A UN MODELO DE DECISIÓN PARA VALORAR ACTIVOS INTANGIBLES BASADOS EN TECNOLOGÍA: CASO ESTUDIO COMPAÑÍA DE SOFTWARE COLOMBIA S.A. ${ }^{1}$
}

\section{UMA APROXIMAÇÃO PARA UM MODELO DE DECISÃO PARA AVALIAR ATIVOS INTANGÍVEIS BASEADA NA TECNOLOGIA: UM ESTUDO DE CASO DA COMPANHIA DE SOFTWARE COLÔMBIA S.A.}

\section{AN APPROXIMATION TO A DECISION MODEL TO VALUATE INTANGIBLE ASSETS BASED ON TECHNOLOGY: CASE STUDY OF COLOMBIA S.A SOFTWARE COMPANY}

\author{
Patricia González González \\ Doutorado em Controladoria e Contabilidade na FEA/USP \\ Professora da Facultad de Ciencias de la Administración Universidad del Valle \\ Sede San Fernando, Edificio 124 \\ Dirección: Cra 56 No. 1 A Oeste 45, Edificio 1, Apto 401 - Cali - Colombia \\ E-mail: pagonza@univalle.edu.co \\ Teléfono: 57251857325130822
}

\section{RESUMEN}

El objetivo de este artículo es proponer un modelo de decisión para valorar activos intangibles basados en tecnología, el cual incorpora los tres métodos de valoración clásicos y aceptados en el SFAS 157: costo, mercado e ingresos. La metodología aplicada fue la de caso estudio y a partir del cual se planteó la necesidad de estimar el valor justo de mercado para un software creado por la Compañía de Software Colombia S.A. Para tal fin fue diseñado un modelo de decisión que involucra los fundamentos teóricos expuestos por Bertolotti (1995) y Reilly (1998). Los resultados obtenidos por cada método permiten, para el caso presentado, establecer el rango entre el cual oscila el valor justo de mercado del software. Se puede concluir que aplicando el modelo

\footnotetext{
${ }^{1}$ Artigo recebido em 29.06.2010. Revisado por pares em 17.10.2010. Reformulado em 18.12.2010. Recomendado para publicação em 21.12.2010 por Ilse Maria Beuren (Editora). Publicado em 31.03.2011. Organização responsável pelo periódico: FURB.
} 
propuesto se calculó el valor del activo intangible basado en tecnología para la Compañía de Software Colombia S.A. Por otro lado, en el proceso de toma de decisión para asignar un valor a dicho activo, no solo se debe tener en cuenta la información cuantitativa que se genera después de aplicar los tres métodos de valoración, sino que también se debe considerar en dicho proceso, aspectos cualitativos como son la selección de la premisa que está orientando al tomador de decisión y el análisis de factores relacionados con clientes, mercado, precio, competencia, tasas de retorno, tasas de capitalización, tasas de participación de utilidades, de regalías, etc.

Palabras claves: Modelo de decisión. Métodos de valoración. Software. Activo intangible.

\section{RESUMO}

O objetivo deste artigo é propor um modelo de decisão para avaliar ativos intangíveis baseados em tecnologia, que inclui os três métodos de avaliação clássicos permitidos pelo SFAS 157: custo, mercado e rendimento. A metodologia da pesquisa utilizada foi o estudo de caso e partir da qual surgiu a necessidade de estimar o valor justo de mercado para um software criado pela Companhia de Software Colômbia S.A. Para tal, foi concebido um modelo de decisão que envolve os fundamentos teóricos apresentados por Bertolotti (1995) e Reilly (1998). Os resultados obtidos em cada método permitem, para o caso apresentado, estabelecer o intervalo que oscila entre o valor justo de mercado do software. Pode-se concluir que a aplicação do modelo proposto calculou o valor dos ativos intangíveis baseados em tecnologia para a Companhia de Software Colômbia S.A. Por outro lado, no processo de decisão para atribuir um valor ao ativo, não só se deve levar em conta a informação quantitativa gerada após a aplicação dos três métodos de avaliação, mas também devem ser considerados no processo os aspectos qualitativos, tais como a seleção da premissa que está orientando o tomador de decisão e as análises de fatores relacionados aos clientes, mercado, preços, concorrência, taxas de retorno, taxas de capitalização, taxas de participação de lucros, royalties, etc.

Palavras-chave: Modelo de decisão. Métodos de avaliação. Software. Activo intangível.

\section{ABSTRACT}

The goal of this article is to propose a decision model to valuate intangible assets based on technology, which incorporates the three classical valuation methods allowed by SFAS 157: cost, market and income. The methodology of the research conducted was case study, from which arose the need of estimating the fair market value of a software created by Colombia S.A. Software Company. For this purpose, a decision model was design which involves the theoretical foundations presented by Bertolotti (1995) and Reilly (1998). The results obtained by each method allow, to the case presented, establish the range that fluctuates among the software fair market value. It can be concluded that applying the proposed model calculated the value of the intangible asset based on technology for the Colombia S.A. Software Company. On the other hand, in the process of decision to assign a value to the asset, not only must be taken into account the quantitative information generated after performed the three methods of valuation, but it should also be considered in this process the qualitative aspects, such as the selection of the premise which is guiding the decision maker and the analysis of factors related to the customers, market, price, competition, return rates, capitalization rates, profit share rates, royalties, etc.

Keywords: Decision model. Valuation methods. Software. Intangible assets. 


\section{INTRODUCCIÓN}

Los cambios en el contexto de los negocios han llevado a que los activos intangibles sean en los días de hoy, los creadores de valor en muchos negocios, especialmente, en los llamados de propiedad tecnológica.

En ese sentido, la participación de los activos intangibles en el valor total de las empresas ha venido ganando importancia en los últimos años, tal como fue registrado en el estudio realizado por la empresa Asset Equity Company, ya que en 1985, el valor en libros de las empresas analizadas equivalía al 50\% del valor de mercado; 15 años después, ese mismo valor en libros representaba tan solo el $20 \%$ del valor de mercado. El $80 \%$ restante era atribuido al valor de los activos intangibles de las empresas.

Según Skyrme (1997), la relación del valor de mercado al valor en libros, para todas las empresas en el Dow Jones Industrial fue de 5,3, mientras que la relación para varias de las empresas con valores significativos en activos intangibles fue de 10, en el año 1997.

Por otro lado, los propietarios de negocios, inversionistas, ingenieros, abogados, el gobierno, entre otros, en la mayoría de los casos no tienen claridad acerca del valor que dicho activo puede tener y mucho menos conocen un método que permita razonablemente estimar su valor, a pesar de la importancia de estos activos en las cifras de las compañías.

Algunas veces, la estimación del valor resulta fácil, pues las normas contables y fiscales suelen orientar en cuanto a la forma como se deben valorar algunos de estos activos intangibles. Pero desafortunadamente, existen situaciones en las cuales la valoración de dichos activos debe atender a otros intereses diferentes de los contables o fiscales, como son los relacionados con la compra o venta y, en este contexto, los criterios y los factores a considerar para su valoración son diferentes.

El objetivo de este artículo es proponer un modelo de decisión para valorar activos intangibles basados en tecnología, el cual incorpora los tres métodos de valoración clásicos y aceptados en el SFAS 157: costo, mercado e ingresos.

\section{REVISIÓN BIBLIOGRAFICA}

\subsection{Razones para valorar el activo intangible basado en la tecnología}

Los activos intangibles deben ser valorados adecuadamente, pues de no hacerlo se pueden generar resultados engañosos afectando de manera directa la elaboración de informes, la administración del negocio y la toma de decisión por parte de los dueños, de un potencial comprador, acreedor u otras partes interesadas (MILLON, 1992; BARTOLOTTI, 1995; REILLY, 1994, 1998; BONTIS et al., 1999; CAÑIBANO et al., 2000; LEV, 2003; ANDRIESSEN, 2004; SENETTI et al., 2004; CHIESA, et al., 2005; MARR, 2007; LING y TANG, 2008).

Entre otras razones que existen para valorar un activo intangible basado en tecnología se encuentran aquellas que tienen que ver con aspectos financieros y de impuestos, tales como: financiamiento para adquisiciones; negocios y acuerdos de compra y venta; evaluación de desempeño del gerente; precio de transferencia entre compañías; evaluación del desempeño del negocio, entre otras (MILLON, 1992; BARTOLOTTI, 1995; REILLY, 1994, 1998; BONTIS et al., 1999; CAÑIBANO et al., 2000; LEV, 2003; ANDRIESSEN, 2004; SENETTI et al., 2004; CHIESA et al., 2005; MARR, 2007; KAPLAN y NORTON, 2008; LING y TANG, 2008).

Por otro lado, Marr (2007) considera que los activos intangibles deben ser medidos, y en este caso, tales mediciones se relacionan con aspectos de cumplimiento de metas y compensaciones. Por lo tanto, los resultados de la medición motivan a los funcionarios a cambiar 
su comportamiento y a ser más productivos y eficaces. Marr (2007) encuentra que la medición de activos intangibles contribuye con la toma de decisiones estratégicas relacionadas con continuos procesos de mejoramiento y aprendizaje.

Lo expuesto permite inferir que para las empresas, con activos intangibles, es perentoria la creación de una metodología y de un sistema de valoración que habilite a los contadores y otros tomadores de decisión a identificar y valorar los activos intangibles, además de poderlos evidenciar de forma clara para fines comerciales ante acreedores, proveedores, inversionistas y el gobierno. Esto en consideración a la importancia que dichos activos tienen en el giro del negocio.

\subsection{La valoración del activo intangible a través de la literatura}

Teóricamente, el activo intangible puede ser definido como un ítem no físico que puede generar beneficios futuros. Generalmente se encuentran representados por las patentes, derechos de autor, formulas y procesos secretos, goodwill, marcas, franquicias, entre otros. Esta descripción es la que se utiliza principalmente en casos de litigios por disputas en contratos, infracciones, daños y situaciones relacionadas con impuestos (CAÑIBANO, 2000; LEV, 2003; TALHA, 2004; CHIESA et al., 2005; QUILLINGAN, 2006; MARR, 2007; IASB 38)

Holzmann (apud CHIESA et al., 2005, p. 6) propone una clasificación en la cual se considera el amplio rango de recursos que cubre el activo intangible, así:

\footnotetext{
a) forma parte de un grupo integrado por otros activos del negocio: personal capacitado, lista de correos, lista de clientes, acuerdos;

b) una unidad económica independiente: patentes, derechos de autor, marcas, Know How tecnológico, diseños tecnológicos, software, entre otros.
}

Bertolotti (1995) identifica entre los principales tipos de propiedad intelectual, las marcas, las patentes, los derechos de autor y diseño, know how, secretos comerciales, software y las bases de datos. Reilly (1998) presenta una lista muy parecida pero agrega a esta última categoría los manuales de procedimiento. Este artículo se centrará en el tipo de activos conocidos como activos intangibles basados en tecnología entre los cuales se encuentran las patentes, el conocimiento de procesos y tecnología, diseños de ingeniería, software y bases de datos.

Por otro lado, la utilización de diversos tipos de tecnología requeridas en los procesos de fabricación, que llevan a procesos de producción cada vez más especializados y las presiones sobre tiempos de entrega y costos, obligan a las compañías a buscar socios que apoyen sus procesos de innovación a partir de activos basados en la tecnología.

La creciente demanda por recursos externos de tecnología que soporten los procesos de innovación en las diversas empresas hace que un nuevo mercado este emergiendo. Por tanto, la tecnología es intercambiada entre diferentes empresas a través de transacciones de compra y venta o como parte de acuerdos de cooperación, tales como joint ventures, alianzas, consorcios, etc. (MILLON, 1992; BERTOLOTTI, 1995; REILLY, 1988, 1994, 1998; BONTIS et al., 1999; MARR, 2003; CHIESA et al., 2005; PELLEGRINO, 2005).

Así mismo, la valoración de estos activos es crítica para los accionistas de la compañía en la medida que ellos necesitan conocer el retorno que están generando los activos en las empresas que han realizado inversiones. Por lo tanto, la información generada de la valoración de los activos intangibles es fundamental para los procesos de toma de decisión.

En consideración a lo anterior se ha propuesto una clasificación de los métodos para valorar los activos intangibles (MUN apud CHIESA et al., 2005), a saber: a) los métodos tradicionales - entre los cuales están los de costo, mercado e ingreso; b) los métodos innovadores - el más representativo es el método de las opciones reales. El artículo abordará los métodos 
tradicionales para valorar activos intangibles basados en tecnología y a seguir se exponen las características más importantes de cada uno de ellos.

\subsubsection{Método de Costo}

El método del costo estima el valor de los activos de tecnología a través de medir los gastos necesarios para crearlos y desarrollarlos. El método se basa en el principio económico de la sustitución y el cual consiste en que un inversionista prudente pagaría hasta un valor igual al costo de crear o adquirir un activo similar. El valor del activo intangible de tecnología está relacionado con la estructura del costo.

Entre las clases de costos más utilizadas para la valoración de los activos intangibles de tecnología están el método de costo de reproducción, el método de reposición o reemplazo, costos de prevención y tendencias en costos históricos.

Según Chiesa et al. (2005), el método del costo presenta las siguientes debilidades:

a) no toma en cuenta los beneficios económicos relacionados con la propiedad y explotación de los activos intangibles;

b) se parte de la presunción de que todo gasto debe generar valor y no necesariamente esto siempre ocurre;

c) el método del costo asume que el nivel de efectividad de la inversión realizada en el pasado será la misma en el futuro. Esta presunción carece de todo sentido, si se tiene en cuenta que existen muchas situaciones en las cuales una inversión puede ser caracterizada por diferentes niveles de eficiencia.

El método se puede aplicar para valorar el activo intangible cuando está en un nivel inicial de desarrollo y por lo tanto su mercado no es muy claro; el nivel de incertidumbre es alto y el conocimiento de los negocios futuros es muy limitado (BERTOLOTTI, 1995; REILLY, 1994,1998; CHIESE et al., 2005; PEREGRINO, 2005;)

\subsubsection{Método del Mercado}

Consiste en comparar el precio por el cual activos similares han sido intercambiados entre compradores y vendedores. En otras palabras, consiste en conocer el precio de mercado de activos similares y con base en ello, se fija su precio. El principio económico que orienta este método de evaluación es el de competencia y equilibrio. En un mercado de libre oferta y demanda dichos factores orientan el precio de todos los bienes y servicios a un punto de equilibrio. Debido a la sencillez del método, este es ampliamente usado (REILLY, 1994, 1998; CHIESE et al., 2005).

El método del mercado presenta las siguientes características (BERTOLOTTI, 1995; REILLY, 1994, 1998; CHIESE et al., 2005; PEREGRINO, 2005):

a) identifica los potenciales contextos que serán utilizados para la comparación de precios, así por ejemplo: industria, participación del mercado, capital de inversión;

b) identifica la información apropiada a partir de la cual cada evaluador debe recoger datos relacionados con la transacción, por ejemplo: el valor por el que la transacción debe ser concluida; una medida económica, por ejemplo: ingresos, márgenes o utilidades netas asociadas con el activo intangible basado en tecnología; o alternativamente, una medida operativa, como por ejemplo, el número de usuarios de la tecnología;

c) calcula el porcentaje entre el valor de la transacción y medida económica u operativa, este ratio es llamado múltiplo. 
Por otro lado, para que el método sea exitoso se requiere que el mercado este activo, es decir que se realice un gran número de transacciones, pues el número es importante, ya que pocas transacciones no son sinónimo de un mercado real. Como también es importante que el mercado sea público, la información tiene que estar disponible y ser de fácil acceso para los usuarios.

Finalmente, la principal debilidad identificada es que no considera las características del comprador y del vendedor, pues se asume que el valor de la transacción es similar, para uno y otro, a partir de esta presunción es que se realiza la comparación (CHIESA et al., 2005)

\subsubsection{Método del Ingreso}

El valor de algún activo puede ser expresado como el valor futuro de los flujos de caja que pueden ser obtenidos por la explotación de una tecnología en especial.

Existen diversas medidas del ingreso económico que podrían dar origen a diversos métodos utilizados para valorar activos intangibles basados en tecnología. Entre estas medidas se pueden considerar: ingresos brutos y netos; ingresos operacionales; ingresos antes de impuestos; ingresos netos después de impuestos; flujos de caja, etc.

Para Chiese et al. (2005), este método es el que permite valorar tecnología de la manera más exacta, ya que considera variables operacionales como: mercado, tamaño, precio, estructura de costo y riesgo. Las cuales son importantes según el tipo de tecnología. No obstante su aplicación podría presentar problemas, pues algunos de los datos de la ecuación a veces resultan difíciles de conseguir.

\subsection{Modelo de decisión para valoración de un activo intangible basado en la tecnología}

Para fines de valorar un activo intangible basado en la tecnología, y a partir de la propuesta realizada por Bertolotti (1995) se proponen tres pasos básicos a ser seguidos con la finalidad de que la valoración se realice de la manera más objetiva y clara:

a) El primer paso conlleva a identificar si el activo que se va a valorar es intangible, para lo cual se debe tener en cuenta lo siguiente:

a) el activo debe ser identificable por sí mismo y se debe diferenciar de otros que son usados en el negocio;

b) su naturaleza no puede ser física;

c) el activo debe ser capaz de producir un beneficio económico futuro;

d) el activo debe estar legalmente protegido.

b) El segundo paso consiste en definir las bases de la valoración. En este paso se identifican tres premisas sobre las cuales una valoración puede ser realizada:

a) valor de existencia relacionado con el principio económico de sustitución - el cual se vincula con las estrategias operacionales, de mercado y financieras que existen en función del activo;

b) valor de mercado relacionado con los principios económicos de competición y equilibrio - para lo cual se debe obtener un activo intangible con un precio igual al que se valora en el mercado;

c) valor de liquidación relacionado con el principio económico de expectativa consiste en el cálculo de un precio de realización en un ambiente de venta restringida.

La selección de alguna de las tres premisas de valoración identificadas va a depender 
de la finalidad que tenga el evaluador, pues esta puede estar relacionada solo con la intención de calcular el costo para fines contables y de impuestos, o para calcular el precio o el valor de mercado para un potencial inversionista.

c) El tercer paso consiste en seleccionar el método que mejor se identifique con la premisa seleccionada (método del costo, de mercado o de ingreso). intangible.

En el Gráfico 1 se exhibe los pasos del modelo propuesto para fines de valorar el activo

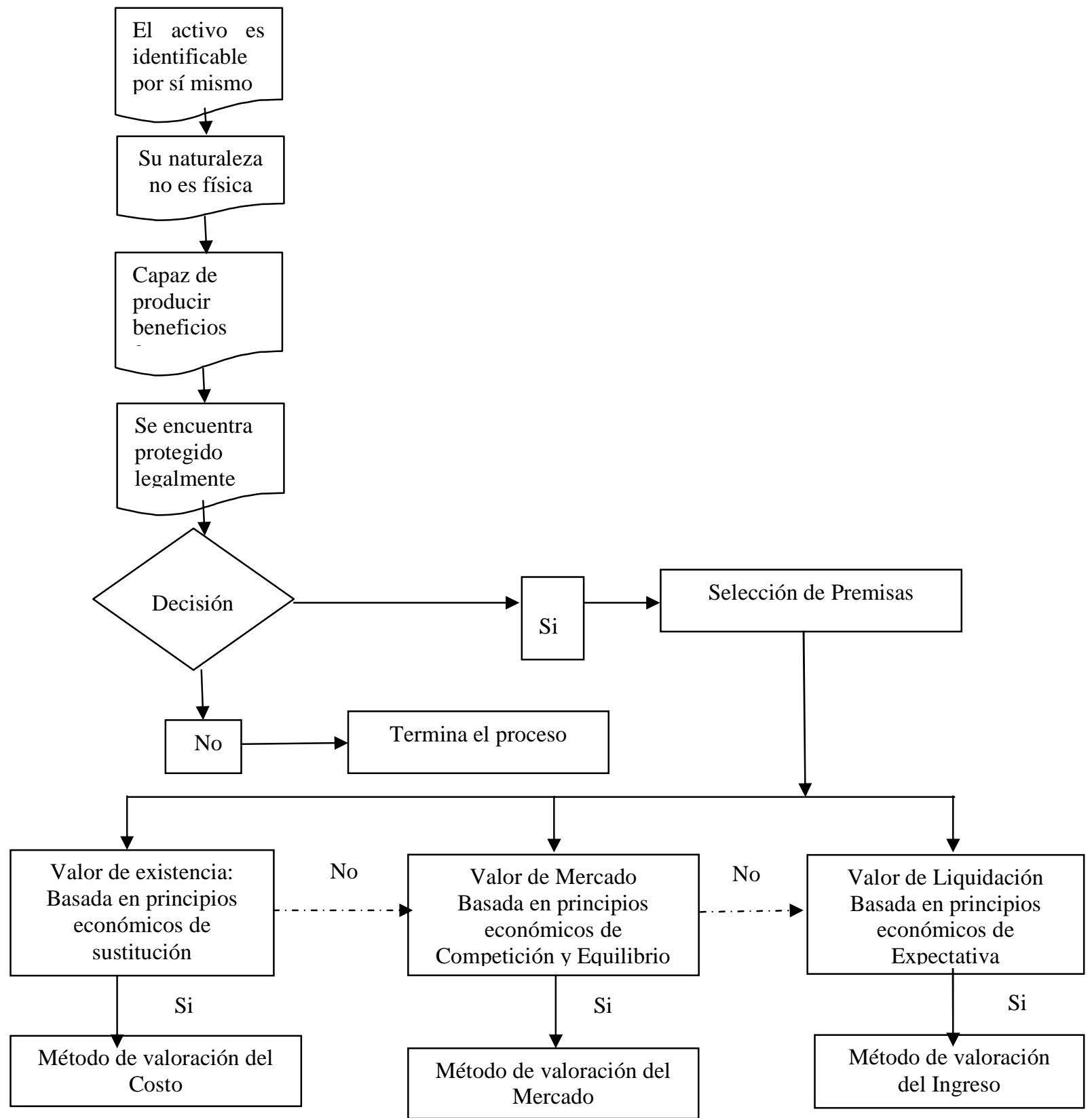

Gráfico 1 - Modelo decisión para valoración de un activo intangible basado en la tecnología 


\subsection{Posición FASB y el IASB sobre el fair value para activos intangibles}

Desde la perspectiva de International Accounting Standard (IAS) 38, los activos intangibles se "identifican como activos no monetarios, sin sustancia física que se mantienen para su uso en procesos de producción o suministro de bienes y servicios, para arrendarlos a terceros o para fines administrativos".

Por otro lado, un activo es un recurso controlado por una empresa como resultado de pasados eventos y del cual se pueden generar beneficios económicos futuros. Del mismo modo, el IAS indica que las empresas frecuentemente gastan recursos o incurren en pasivos en la adquisición, mantenimiento o mejora de recursos intangibles tales como marcas, títulos publicados, etc.).

Otros ejemplos de activos intangibles son: propiedad intelectual, conocimiento técnico; lista de clientes; patentes: derechos de autor; Software; licencias y el diseño e implementación de nuevos procesos.

Es de anotar que existen algunos bienes intangibles que no caben en la definición que es dada por el IAS 38. Una vez que, el IAS exige que un activo intangible sea identificable o separado para diferenciarlo claramente del Goodwill.

Cuando se habla de separarlo se está haciendo referencia a que se pueda demostrar que el beneficio económico futuro generado por dicho activo se puede originar del alquiler, venta, intercambio o distribución, sin que ello implique la necesidad de disponer de los beneficios económicos futuros de otros activos usados en las mismas actividades generadoras de ganancias.

Finalmente, el IAS 38 define el control sobre el recurso "como el poder para disponer de los beneficios económicos futuros, excluyendo a otros individuos de la explotación de aquellos beneficios".

Los beneficios económicos futuros pueden resultar a partir de la venta de un producto, la prestación de un servicio, como también, por la disminución de costos o por el uso adecuado del activo.

Por otro lado, el Financial Accounting Standards Board (FASB) presenta los Staments of Financial Accounting Standards (SFAS) 141, 142, 157 en los cuales se dan elementos suficientes para valorar y contabilizar activos intangibles.

El SFAS 141, tiene que ver con la forma de lidiar con los activos intangibles que resultan de la Combinación de Negocios y el SFAS 142 aborda, el asunto del Goodwill y otros activos intangibles. En el SFAS 157 se aceptan los métodos de mercado, costo e ingreso, como medios para calcular el fair value de los activos.

Es de anotar que estos SFAS fueron diseñados para (TALHA, 2004, p. 29):

a) "mejorar la comparabilidad de la información financiera contenida en los estados financieros;

b) evidenciar de la mejor forma las inversiones realizadas en entidades adquiridas.

c) proveer la más completa información financiera, y;

d) promover la armonización internacional".

No obstante se reconoce que en el campo de las finanzas podríamos encontrar una diversidad de métodos y técnicas que complementan o soportan los tres métodos propuestos y que contribuyen con la valoración de los activos intangibles.

Realizado esta breve revisión conceptual se presenta un caso estudio en el que se hará un análisis comparativo de los resultados que se obtienen al valorar un activo intangible basado en tecnología a partir de los tres métodos propuestos. 


\section{METODOLOGIA}

La metodología empleada se circunscribe en el desarrollo de un caso estudio, en el que se presenta el modelo de decisión creado a partir de las propuestas realizadas por Bertolotti (1995) y Reilly (1998).

A partir de caso estudio se planteó la necesidad de estimar el valor justo de mercado para un software creado por la Compañía de Software Colombia S.A.

\section{CASO ESTUDIO: COMPAÑÍA DE SOFTWARE COLOMBIA S.A.}

La Compañía de Software Colombia S.A. es una empresa colombiana dedicada al diseño y soporte de aplicativos para fines gerenciales. Su participación en el mercado nacional representa el $60 \%$ y emplea un total de 200 personas a nivel nacional entre programadores, diseñadores, técnicos y personal administrativo. A causa de sus desarrollos y confiabilidad de sus productos, goza de credibilidad en el mercado nacional y por lo tanto pretende entrar a nuevos mercados en la región.

En la actualidad, la empresa está trabajando en el diseño de un nuevo aplicativo que permite integrar datos de la contabilidad financiera con el diseño de un sistema gerencial que parte de principios del Cuadro de Mando Integral y el Time Drive ABC. Este aplicativo pretende que el gerente en tiempo real pueda medir el logro de sus estrategias y realizar un continuo seguimiento a las mismas, como también, le permite medir el costo de las actividades en los procesos de la organización.

El gerente de la Compañía de Software Colombia S.A. recurrió a un experto en valoración de activos intangibles basados en tecnología para que calcule el valor de este aplicativo, de tal manera que se pueda fijar un precio que sea competitivo para el mercado, en el próximo año. Para tal fin, el experto realizó una simulación a partir de los tres métodos que conoce para valorar activos intangibles y calcular, así, el valor para el software.

\subsection{Información para valorar activos de tecnología}

El consultor basado en la propuesta metodológica hecha por Reilly (1998), para valorar activos de tecnología, identifica la información necesaria para su análisis.

\subsubsection{Método de costo}

a) Los cálculos se hicieron tomando como base el abordaje de ahorros en costos por investigación y desarrollo;

b) Debido a que la Compañía de Software Colombia S.A. ha mejorado sus procesos tecnológicos y después de un estudio realizado, se encontró que esta empresa ha generado unos ahorros en costos de investigación y desarrollo en un $15 \%$, comparado con los de la competencia:

Costos promedio por investigación y desarrollo de la competencia:.................................. 20\% de los ingresos proyectados.

Costos promedio por investigación y desarrollo de la Compañía Software de Colombia S.A.....................................5\% de los ingresos proyectados.

Total ahorro en costos por investigación y desarrollo de la Compañía de Software Colombia S.A...................................15\% de los ingresos proyectados. 


\subsubsection{Método de ingreso}

a) Para calcular el valor del activo intangible basado en tecnología por el método del ingreso, se consideró el abordaje de participación de utilidades;

b) La Compañía de Software Colombia S.A. tiene un acuerdo de repartición de regalías con un distribuidor de sus productos, en los siguientes términos: el 25\% de los ingresos proyectados en ventas de los productos le corresponden a la Compañía Software de Colombia S.A. y el $75 \%$ de esos ingresos le corresponden a su distribuidor;

c) El experto estimó que los gastos de operación representan el $70 \%$ de los ingresos proyectados;

d) Dentro del análisis también se consideró el costo que generan los activos que no son tecnología y que contribuyen con la generación de ingresos, los cuáles se estima ascienden a $\$ 10.000$ (depreciación, mantenimiento, mejoras, etc.).

\subsubsection{Método del mercado}

a) Para fines de estimar el valor del activo intangible basado en tecnología por el método de mercado, el experto utilizó el abordaje de cálculo de regalías;

b) El asesor después de realizar un análisis de los acuerdos de licencias en otras empresas similares a la Compañía de Software Colombia S.A., concluyó que la tasa de regalías por el uso de licencias se encuentra en un rango que oscila entre un 15\% y $20 \%$ de los ingresos proyectados.

\subsubsection{Información común para los tres casos}

a) Los ingresos proyectados para el próximo año son: $\$ 500$ millones;

b) La tasa de impuesto: $35 \%$;

c) Tasa de capitalización del producto: $20 \%$ (esta tasa corresponde a una tasa de retorno sobre la inversión, que para este caso, se estimó en $20 \%$ ).

A seguir el experto procede a realizar los cálculos bajo los tres métodos sugeridos para calcular el valor del software.

\subsection{Desarrollo del caso}

\subsubsection{Aplicación del modelo de decisión}

El primer paso consiste en identificar si el activo que se va a valorar es intangible, como se muestra en lo Gráfico 2. 


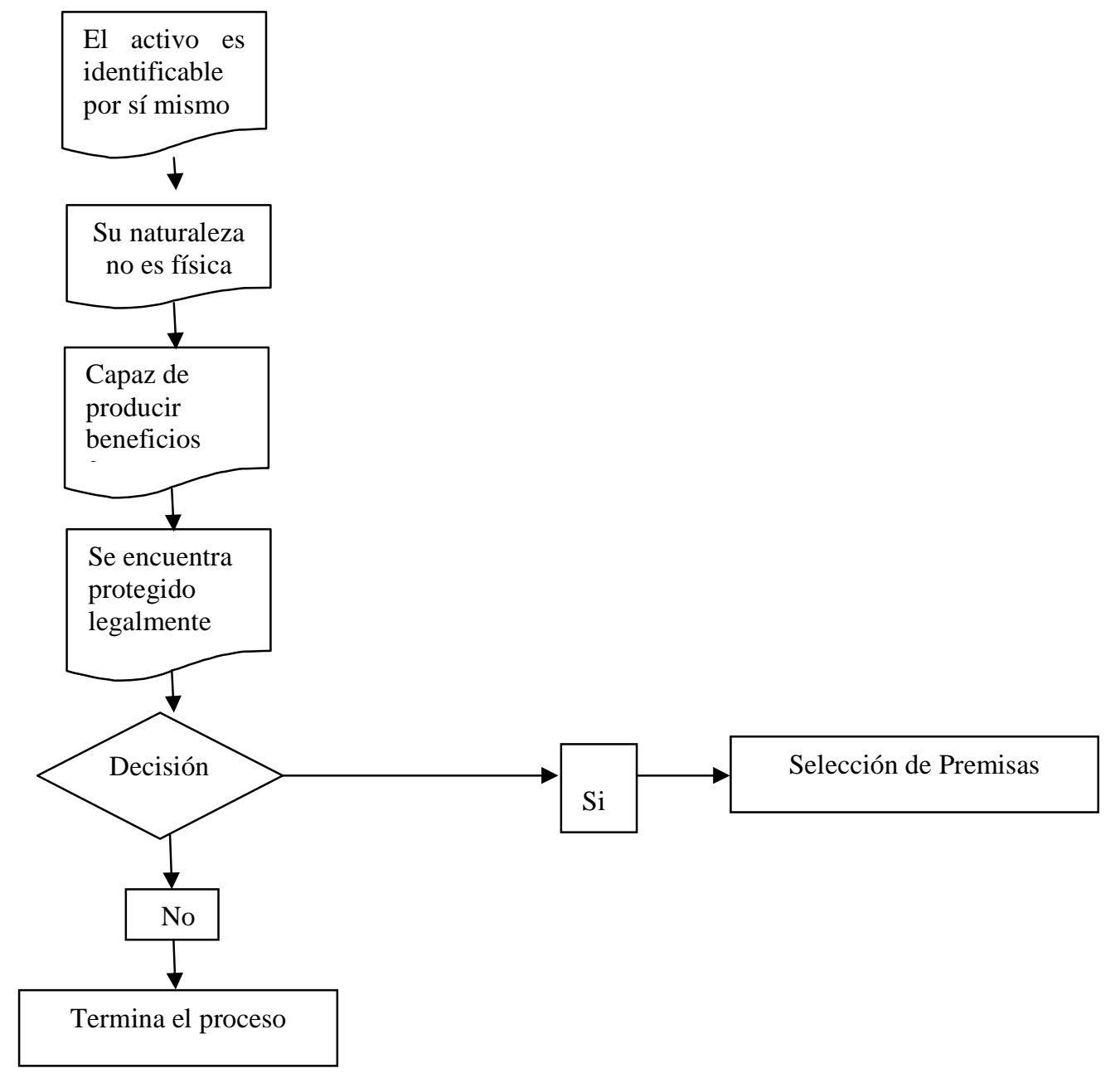

\section{Grafico 2 - Pasos a seguir para identificar si el activo a valorar es intangible}

El segundo paso consiste en definir las bases de la valoración, una vez que en el primer paso se confirmó que el activo a ser valorado es intangible. En este paso se identifican tres premisas sobre las cuales una valoración puede ser realizada, como se muestra en lo Gráfico 3.

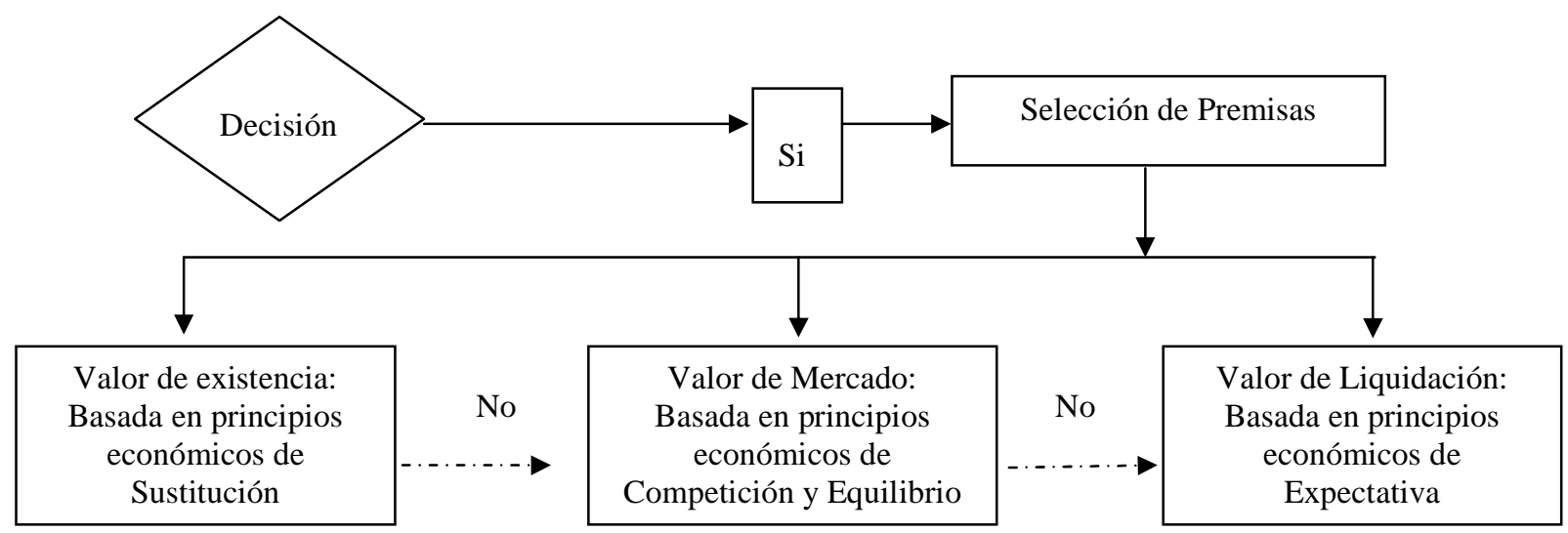

Gráfico 3 - Identificación de premisas para la valoración del activo 
El tercer paso consiste en seleccionar el método que mejor se identifique con las premisas presentadas en el paso 2, como se muestra en lo Gráfico 4.

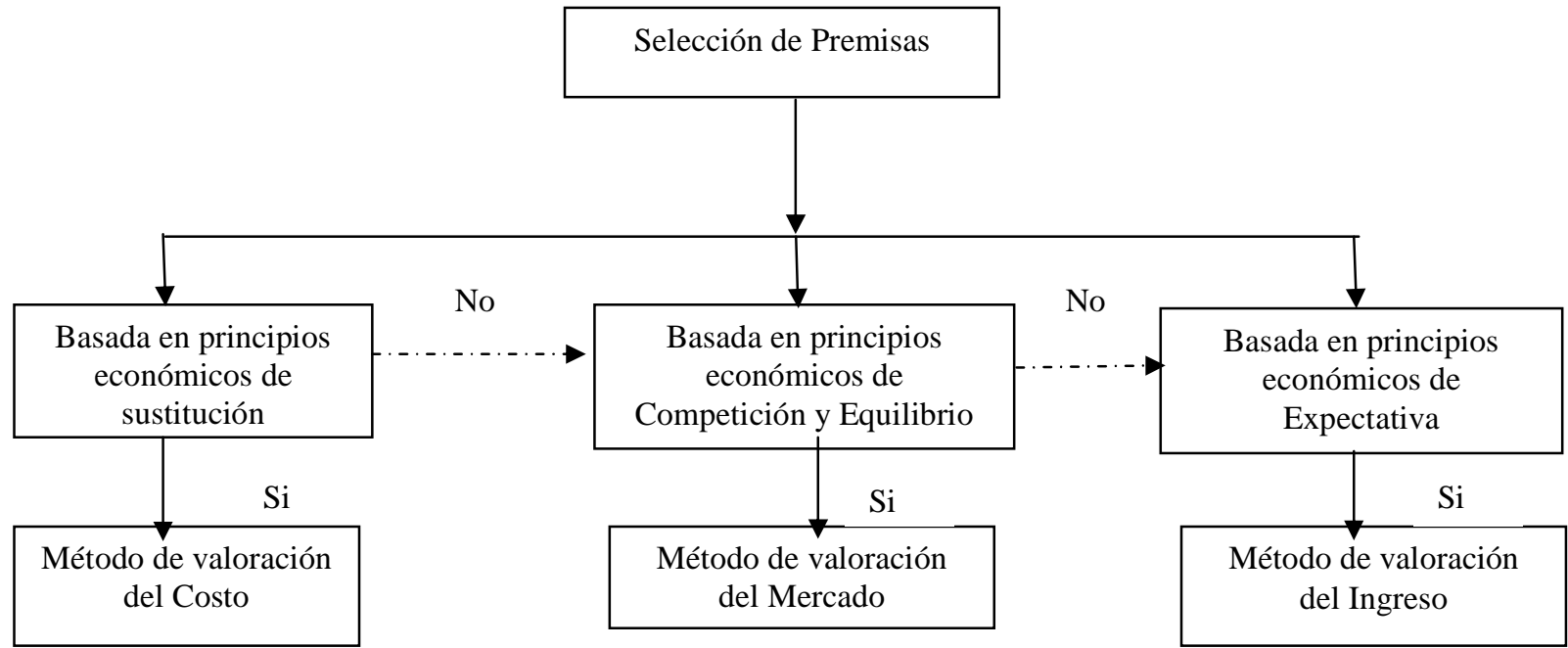

Gráfico 4 - Selección de método conforme las premisas identificadas

\subsubsection{Cálculos de los valores justos de mercado}

En la literatura (SFAS 157) se hace referencia a los métodos del costo, mercado e ingreso

a) Método del costo con base en un abordaje de ahorros en costos por investigación y desarrollo (Cuadro 1).

\begin{tabular}{|l|r|}
\hline \multicolumn{1}{|c|}{ Variables a ser consideradas } & Año a ser proyectado 20X1 \\
\hline Ingresos proyectados & $\$ 500.000$ \\
\hline Porcentaje del ahorro en costos por I+D & $15 \%$ \\
\hline Incremento de los ingresos por ahorro en costos por I+D & $\$ 75.000$ \\
\hline Menos Impuestos sobre la renta 35\% & $\$(26.250)$ \\
\hline Incremento Neto de los ingresos después de impuestos & $\$ 48.750$ \\
\hline Tasa de retorno & $20 \%$ \\
\hline Valor del Software & $\$ 243.750$ \\
\hline Potencial valor justo del mercado & $\mathbf{\$ 2 4 3 . 7 5 0}$ \\
\hline
\end{tabular}

Cuadro 1 - Método del costo

b) Método de mercado con base en un abordaje de regalías (Cuadro 2).

\begin{tabular}{|l|r|r|}
\hline \multicolumn{1}{|c|}{ Variables a ser consideradas } & Año a ser proyectado 20X1 \\
\hline Ingresos Proyectados & $\$ 500.000$ & $\$ 500.000$ \\
\hline Porcentaje del Rango de las Tasas de Regalías & $15 \%$ & $20 \%$ \\
\hline Regalías anuales proyectadas & $\$ 75.000$ & $\$ 100.000$ \\
\hline Menos Impuesto sobre la renta 35\% & $(26.250)$ & $(35.000)$ \\
\hline Regalías netas anuales proyectadas & 48.750 & 65.000 \\
\hline Tasa de retorno & $20 \%$ & $20 \%$ \\
\hline Valor del Software & $\$ 243.750$ & $\$ 325.000$ \\
\hline Potencial valor justo de mercado & & $\$ 325.000$ \\
\hline
\end{tabular}

Cuadro 2 - Método de mercado 
c) Método del ingreso desde un abordaje de la asignación de las utilidades entre el creador de la licencia y quien la distribuye o usufructúa (Cuadro 3).

\begin{tabular}{|c|c|c|}
\hline Variables a ser consideradas & \multicolumn{2}{|c|}{ Año a ser proyectado 20X1 } \\
\hline Ingresos Proyectados & & $\$ 500.000$ \\
\hline Gastos de Operación $70 \%$ & & $(\$ 350.000)$ \\
\hline Ingresos antes de impuestos & & $\$ 150.000$ \\
\hline Menos Impuesto sobre la renta $35 \%$ & & $(\$ 52.500)$ \\
\hline Menos cargos por uso de Activos Tangibles & & $(10.000)$ \\
\hline Ingreso Económico Proyectado & & $\$ 87.500$ \\
\hline \% de Repartición de utilidades & Titular de la licencia & $\begin{array}{l}\text { Quien está autorizado } \\
\text { para explotarla }\end{array}$ \\
\hline & $25 \%$ & $75 \%$ \\
\hline & $\$ 21.875$ & $\$ 65.626$ \\
\hline Dividido por la tasa de capitalización & $20 \%$ & $20 \%$ \\
\hline Valor del software & $\$ 109.375$ & $\$ 328.125$ \\
\hline Potencial valor justo de mercado & & $\$ 328.125$ \\
\hline
\end{tabular}

Cuadro 3 - Método del ingreso

\subsection{Análisis de los resultados}

Los resultados obtenidos, conforme los abordajes seleccionados para cada uno de los métodos (Gráfico 5), fueron los siguientes:

a) Método de Costo \$ 243.500;

b) Método de Mercado \$325.000;

c) Método de Ingreso \$328.125.

Dentro del enfoque de Reilly (1998), el valor final del software debería ser un número que podría estar en el rango de valores que cada uno de los métodos arrojó. Por lo tanto para este caso, el gerente podría fijar un intervalo entre el menor y mayor valor obtenido (menor $\$ 243.750$ y mayor valor \$328.125). De esta manera el valor justo de mercado para el software estaría oscilando entre estos dos valores.

Del mismo modo Really (1998) considera otra opción y es la de tomar alguno de los valores obtenidos en los tres métodos y ese sería el valor del software. Para lo cual sería importante tener en cuenta la premisa de selección que orienta cada método (tal como se encuentran representadas en el Gráfico 1) y que depende de la preferencia del tomador de decisión. Con base en ello se escoge el valor que se le asignará al activo intangible, así por ejemplo, si la premisa que orienta la decisión está basada en principios económicos de sustitución (que corresponde al método de costo), entonces el valor del software sería de \$243.750, esto por citar un ejemplo.

Finalmente, Really (1998) considera que el valor de una tecnología debería ser aquel que resulte del juicio del analista a partir de tener en cuenta todos los factores relevantes y del pesaje imparcial de todas las evidencias disponibles que lanza el mercado. Lo que significa que la información generada por los métodos se debe complementar con otros aspectos de carácter cualitativo y cuantitativo, tal es el caso de información sobre clientes, mercado, precio, competencia, tasas de capitalización, tasas de participación de utilidades, de regalías, etc. 


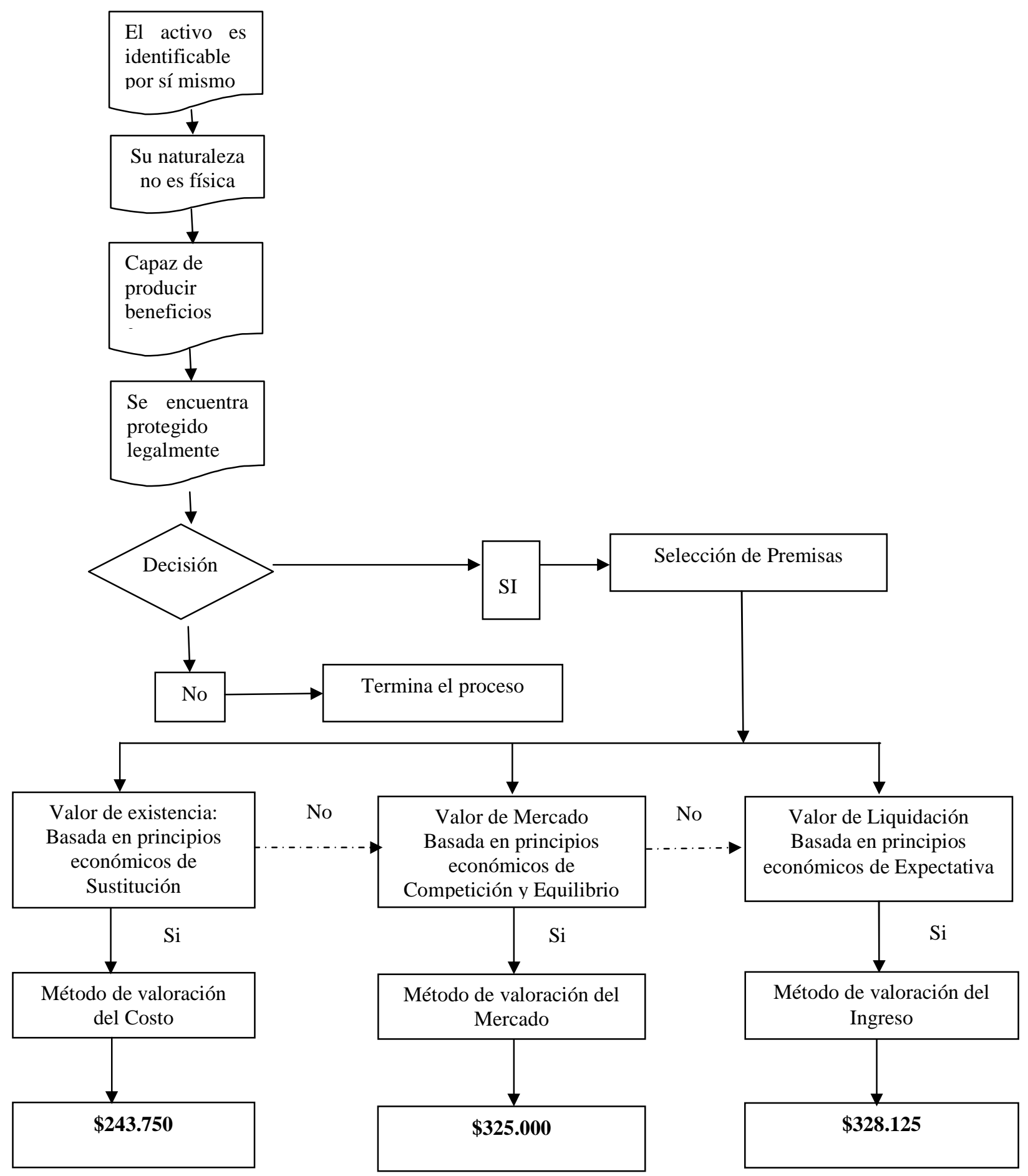

Propuesta de valor justo de mercado por cada método

Gráfico 5 - Resultados obtenidos por cada método 


\section{CONCLUSÕES}

La importancia de la valoración de activos intangibles basados en tecnología es cada vez más preponderante, pues son muchos los negocios en los días de hoy que giran en torno a fabricar productos o prestar servicios de carácter tecnológico.

La manera de medirlo evidentemente repercutirá en la buena asignación de un precio final, el cual debe ser competitivo, pues de no ser así, la continuidad del negocio puede estar en un alto riesgo.

Tal como se pudo apreciar en el caso estudio presentado, los métodos de costo, mercado e ingreso, empleados adecuadamente nos permiten calcular un valor para un activo intangible como es el caso de un software.

Finalmente, el valor del activo intangible basado en tecnología debe ser el resultado de un análisis juicioso de todos los factores que son relevantes y de dar un peso de manera imparcial a cada una de las variables que el mercado ofrece, para este tipo de análisis.

\section{REFERENCIAS}

ANDRIESSEN, Daniel. IC valuation and measurement: classifying the state of the art. Journal of Intellectual Capital, p. 230-242, 2004. 10.1108/14691930410533669

BERTOLOTTI, Nick. Valuing intelectual property. Managing Intellectual Property, p. 28.32, 1995.

BONTIS, Nick; DRAGONETTI, Nicola C.; JACOBSEN, Kristine; ROOS, Göran. The knowledge toolbox: a review of the tools available to measure and manage intangible Resources. European Management Journal, v. 17, n. 4, p. 391- 401, Aug., 1999. doi:10.1016/S02632373(99)00019-5

CANIBAÑO, Leandro; GARCIA-AYUSO, Manuel; SANCHEZ, Paloma. Accounting for intangibles: a literature review. Journal of Accounting Literature, v. 19, p. 102-130, 2000.

CHIESA, Vittorio; GILARDONI, Elena; MANZINI, Raffaella. The valuation of technology in buy cooperate-sell decisions. European Journal of Innovation Management, v. 8, n. 1, p. 5-30, 2005. doi:10.1108/14601060510578556

FINANCIAL ACCOUNTING STANDARD BOARD (FASB). Disponible en: <http://asc.fasb.org/home>. Acceso en: 3 nov. 2010.

INTERNATIONAL FINANCIAL REPORTING STANDARDS (IFRS). Disponible en: <http://www.ifrs.org>. Acceso en: 3 nov. 2010.

LEV, Baruch. Remarkson the measurement, valuation and reporting of intangible assets. Economic Policy Review, p. 17-22, 2003.

LIN, Grace; TANG, Jerry. Appraising intangible assets from the viewpoint of value drivers. Journal of Business Ethics, p.679-689, 2009. doi:10.1007/s10551-008-9974-y

MARR, Bernard. Measuring and managing intangible value drivers. Business Strategy Series, v. 8, n. 3, p. 172-178, 2007. doi:10.1108/17515630710684169

MILLON, Tom. Computer software valuation. Don't be led astray by a quick approach. The National Public Accounting. p. 14-17, 1992.

PELLEGRINO, Mike. Valuation of embedded software. Valuation Strategies, p. 12- 49, 2005. 
QUILLIGAN, Laura. Intangible Assets identification and valuation under IFRS 3. Accountancy Ireland. v. 38, n. 3, p.10-12, 2006.

REILLY, Robert. The valuation of intangible assets and intellectual propert. Finanacial Managers Statements, p. 50-56, 1988.

REILLY, Robert. Valuation of intangible assets for bankruptcy and reorganization purpose. Ohio CPA Journal, p. 25-30, 1994.

REILLY, Robert. The Valuation of proprietary technology. Management Accounting. p. 45-49, 1998.

SENNETTI, John T.; KIM, Changsoo; SELLANI, Robert. Measuring the effect of investment in Intellectual Capital. Journal of Applied Management and Entrepreneurship. v. 9, n. 2, p. 8296, 2004.

TALHA, Mohamed. Valuation of intangible assets in accounting. Construction Accounting \& Taxation. p. 25-31, 2004. 